\title{
Evaluación de lesiones orales en pacientes intubados y su asociación con el motivo de hospitalización.
}

\author{
Evaluation of oral lesions in intubated patients and its \\ association with the reason of hospitalization.
}

\author{
Lizett Trujillo Morales,* Rubén Valenzuela Esquivel,‡ Karla L Tovar Carrillo,§ Juan Carlos Cuevas Gonzales, \\ Alejandro Donohue Cornejo, ${ }^{\S}$ Salvador David Nava Martínez, ${ }^{\S}$ León Francisco Espinosa Cristóbal ${ }^{\S}$
}

RESUMEN

Introducción: La intubación es un procedimiento que permite la asistencia ventilatoria cuando la vía aérea y la consciencia están comprometidas. Los elementos utilizados en este procedimiento se encuentran en contacto directo con estructuras dentofaciales, causando diversos tipos de lesiones, principalmente bucales. Aunque existen cuidados clínicos durante procesos de intubación, hay poca información, particularmente de la zona norte del país donde se hayan evaluado las posibles asociaciones entre los motivos de consulta más frecuentes y las diversas características, tanto clínicas como no clínicas de pacientes que han sido intubados. Objetivo: Identificar las alteraciones bucodentales más frecuentes en pacientes intubados, así como explorar las posibles asociaciones de acuerdo con los motivos de intubación más frecuentes. Material y métodos: Se realizó un estudio observacional, transversal y comparativo en el cual se evaluaron alteraciones bucodentales y sistémicas de pacientes intubados. Los grupos de estudio se desarrollaron de acuerdo con el motivo de intubación y la determinación de las asociaciones fue con cada una de las alteraciones bucodentales y sistémicas. Resultados: El motivo de intubación más frecuente fue el evento cerebral vascular (EVC) y las alteraciones dentofaciales más prevalentes fueron caries, lengua saburral y cálculo dental, entre otras. Además, se encontraron diferencias significativas entre pacientes con EVC, mostrando una mayor frecuencia en tabaquismo, hipertensión arterial y diabetes mellitus, así como en la presencia de gingivitis y úlceras. Pacientes con traumatismo craneoencefálico (TCE) tuvieron mayor frecuencia en la presencia de periodontitis. Conclusión: El motivo de hospitalización y las condiciones sistémicas preexistentes pueden ser

\section{ABSTRACT}

Introduction: Intubation is a procedure that allows ventilatory assistance when the airway and consciousness are compromised. The elements used in this procedure are in direct contact with dentofacial structures causing various types of injuries, mainly oral. Although there is clinical care during intubation processes, there is little information, particularly from the northern part of the country where the possible associations between the most frequent reasons for consultation and the various clinical and non-clinical characteristics of patients who have been intubated have been evaluated. Objective: The objectives of the present study were to identify the most frequent oral alterations in intubated patients, as well as to explore possible associations according to the most frequent reasons for intubation. Material and methods: An observational, cross-sectional and comparative study was carried out in which oral and systemic alterations of intubated patients were evaluated. The study groups were formed according to the reason for intubation and the association was determined with each of the oral and systemic disorders. Results: The most frequent reason for intubation was the vascular cerebral event (CVA) and the most prevalent dentofacial alterations were caries, coated tongue, and dental calculus, among others. In addition, significant differences were found between patients with CVA, showing a higher frequency in smoking, hypertension, and diabetes mellitus, as well as in the presence of gingivitis and ulcers. Patients with traumatic brain injury (TBI) had a higher frequency in the presence of periodontitis. Conclusion: The reason for hospitalization and pre-existing systemic conditions can be a risk factor for developing specific oral lesions before and during the intubation period.

\footnotetext{
* Alumna de la Maestría en Ciencias Odontológicas. Facultad de Odontología.

‡ Departamento de Estomatología.

§ Profesor Investigador del Departamento de Estomatología. Instituto de Ciencias Biomédicas.
}

Universidad Autónoma de Ciudad Juárez. Ciudad Juárez, Chihuahua, México.

Recibido: 09 de junio de 2021. Aceptado: 16 de septiembre de 2021.

Citar como: Trujillo ML, Valenzuela ER, Tovar CKL, Cuevas GJC, Donohue CA, Nava MSD et al. Evaluación de lesiones orales en pacientes intubados y su asociación con el motivo de hospitalización. Rev ADM. 2021; 78 (5): 251-257. https://dx.doi.org/10.35366/102031 
un factor de riesgo para desarrollar lesiones bucales específicas antes y durante el periodo de intubación.

Palabras clave: Intubación, complicaciones, alteraciones orales, alteraciones sistémicas, odontología.
Keywords: Intubation, complications, oral alterations, systemic alterations, odontology.

\section{INTRODUCCIÓN}

$\mathrm{L}$ a intubación es un procedimiento que permite la asistencia ventilatoria durante un procedimiento de anestesia o a pacientes que requieren ventilación mecánica por encontrarse en cuidados intensivos. ${ }^{1}$ La intubación suele ser necesaria por aquellas situaciones que provocan alteraciones en la normalidad de la función respiratoria, se podría resumir en los siguientes casos: 1) parada cardiorrespiratoria; 2) protección de la vía aérea; 3) traumatismo craneoencefálico donde la vida del paciente se encuentre en riesgo; 4) paciente con insuficiencia respiratoria aguda; y 5) nivel de consciencia bajo y una puntuación en la escala de Glasgow menor a 8 puntos. ${ }^{2}$

Es bien sabido que la presencia de los tubos utilizados en los procedimientos de intubación se encuentran en contacto directo con estructuras de la vía aérea superior e inferior, las cuales pueden causar diversos tipos de lesiones, principalmente en la mucosa derivado de periodos prolongados o traumáticos primordialmente en el uso de tubos de alto volumen y presiones excesivas en el manguito del tubo endotraqueal. ${ }^{1}$ Por otro lado, la incidencia de lesiones dentales ocasionadas por intubación durante procedimientos donde se utilizó anestesia general es de 1 en 1,501 a 1 en 1,000, mientras que la distribución de las lesiones orales más frecuentes fueron desde luxaciones, movilidad dental, fractura dental hasta daños a prótesis dentales. ${ }^{3}$ Diversas investigaciones han determinado que los factores de riesgo para la lesión dental durante la intubación son la apertura bucal limitada, movilidad mandibular limitada, baja visibilidad en la hipofaringe así como la poca movilidad del cuello; ${ }^{4}$ sin embargo, otras condiciones propias del paciente podrían también involucrarse en el desarrollo de lesiones bucales durante la intubación. Además, la presencia de caries dental, periodontitis, restauraciones insuficientes, la existencia de restauraciones cerámicas, entre otras, asociadas con la intubación podrían generar significativamente un mayor riesgo de lesiones bucodentales. ${ }^{5}$ Es por ello que el personal de salud debe contar con el conocimiento básico de la normalidad y anormalidad de las estructuras de la cavidad oral para reconocer los riesgos y las medidas preventivas para evitar generar complicaciones durante el proceso de intubación. ${ }^{4}$

Es probable que en muchas instituciones de salud no se tenga un registro de alteraciones bucodentales ocasionados durante el proceso de intubación, de esta manera se cree que dichas lesiones se presentan por la condición sistémica del paciente, por encontrarse bajo intubación por vía orotraqueal o nasotraqueal, así también por la falta de cuidados y capacitación del personal para llevar un correcto control e higiene oral en estos pacientes. Los objetivos del presente estudio fueron identificar las alteraciones bucodentales más frecuentes en pacientes intubados, así como explorar las posibles asociaciones de acuerdo con los motivos de intubación más frecuentes.

A pesar de la diversa literatura relacionada con las alteraciones causadas por la intubación, no existe información suficiente particularmente de la zona norte del país que hayan evaluado ni explorado las posibles asociaciones entre los motivos de consulta más frecuentes y las diversas características, tanto clínicas como no clínicas, de pacientes que han sido intubados.

\section{MATERIAL Y MÉTODOS}

Se realizó un estudio observacional, transversal y descriptivo. Se estudiaron a 50 pacientes entre 17 y 50 años sometidos a un proceso de intubación orotraqueal en el Hospital General de Ciudad Juárez, Chihuahua del Instituto Chihuahuense de Salud. El estudio fue aprobado por el Jefe del Departamento de Enseñanza de la institución. Se incluyeron pacientes con patologías que requirieran manejo avanzado de la vía aérea; los criterios de exclusión fueron pacientes con procedimientos quirúrgicos muy invasivos, alto nivel de complejidad clínica y alto riesgo de infección. Se realizó una recolección de datos a través de expedientes clínicos, y con apoyo de los familiares directos, debido a que se encontraban inconscientes o sedados. Con el apoyo del personal hospitalario, un odontólogo general realizó exploraciones faciales y bucodentales y se llevó un registro de las lesiones presentes. Los grupos de estudio se realizaron de acuerdo con el motivo de intubación más frecuente: 1) pacientes con 
traumatismo craneoencefálico (TCE); y 2) pacientes con evento cerebral vascular (EVC).

Análisis estadístico. Se aplicó estadística descriptiva donde los datos se calcularon en frecuencias y promedios. El análisis entre grupos se realizó con el análisis de $\chi^{2}$ y las diferencias entre los grupos fueron determinadas cuando se obtuviera un valor de $p<0.05$. Se utilizó el programa estadístico SPSS Statistics versión 25.

\section{RESULTADOS}

Se evaluaron 50 pacientes, generalmente adultos intubados y atendidos en el Hospital General de Ciudad Juárez como se describe en la Tabla 1. Se presenta la distribución general de acuerdo con el género, se encontró que los sujetos varones fueron más frecuentes $(66 \%)$ que las mujeres (34\%) con edades muy similares entre ellos (49.3 \pm 17.1 años). Por otra parte, las condiciones de hospitalización más frecuente fueron pacientes por evento cerebral hemorrágico (32\%), seguido de traumatismo craneoencefálico (24\%), neumonía (6\%), intoxicación por drogas (4\%), mientras que los pacientes restantes fueron intubados por otras condiciones, como neumonía, intoxicación por drogas, abdomen agudo, acidosis metabólica, cáncer pulmonar, colecistitis crónica litiásica, EPOC, fracturas múltiples/choque hipovolémico, heridas por arma blanca de tórax, insuficiencia cardiaca congestiva, meningitis, pancreatitis, perforación de víscera hueca, taponamiento cardiaco, tuberculosis, glioma cerebral, Guillain-Barré, herida por arma de fuego en tórax y lesión medular de baja frecuencia, las cuales fueron consideradas dentro de esta última variable (34\%). Asimismo, gran cantidad de casos negaron la existencia de antecedentes heredofamiliares (78\%), seguido del antecedente de madre diabética $(8 \%)$ y con padres diabéticos $(6 \%)$. Adicionalmente, un poco menos de la mitad negaron presencia de antecedentes patológicos (44\%), seguido de la presencia de hipertensión arterial (22\%), hipertensión arterial y diabetes mellitus (12\%), diabetes mellitus (10\%) y con arritmia cardiaca (4\%). Lo anterior sugiere que los sujetos involucrados en nuestra población tienen diversas características sociodemográficas y clínicas que hacen una muestra heterogénea.

En la Tabla 2 se muestra la distribución de las alteraciones bucales de los pacientes intubados. Estos resultados podrían sugerir que la frecuencia de las alteraciones bucales se encontraría asociada con enfermedades orales crónicas, como lo son la caries dental $(76 \%)$, periodontitis (44\%) y cálculo dental (58\%), pero también alteraciones bucales como la lengua saburral (64\%), xerostomía (52\%) y úlceras (36\%) podrían estar asociadas con la presencia de aditamentos físicos que promuevan un riesgo para el desarrollo de éstas (Figura 1).

La Tabla 3 muestra la distribución de alteraciones generales y bucales de casos que sufrieron un evento cerebral vascular o traumatismo craneoencefálico. De manera general, los sujetos con eventos cerebrales vasculares tuvieron mayor presencia de hábitos y alteraciones sistémicas y bucales, como lo fue el tabaquismo (56.3\%), hipertensión arterial (50\%), hipertensión arterial con diabetes mellitus (37.5\%), gingivitis (37.5\%), fractura (12.5\%),

\section{Tabla 1: Distribución general de la población, condiciones de hospitalización personales $(\mathrm{N}=50)$.}

\begin{tabular}{|c|c|}
\hline & n (\%) \\
\hline Edad* & $49.3 \pm 17.1$ \\
\hline \multicolumn{2}{|l|}{ Sexo } \\
\hline Hombre & $33(66)$ \\
\hline Mujer & $17(34)$ \\
\hline \multicolumn{2}{|l|}{ Condiciones de hospitalización } \\
\hline Evento cerebral vascular & $16(32)$ \\
\hline Traumatismo craneoencefálico & $12(24)$ \\
\hline Neumonía & $3(6)$ \\
\hline Intoxicación por drogas & $2(4)$ \\
\hline Otras causas & $17(34)$ \\
\hline \multicolumn{2}{|l|}{ Antecedentes heredofamiliares } \\
\hline Negados & $39(78)$ \\
\hline Madre diabética & $4(8)$ \\
\hline Padres diabéticos & $3(6)$ \\
\hline Madre finada con cáncer de mama & $1(2)$ \\
\hline Padre con cáncer de próstata & $1(2)$ \\
\hline Padre hipertenso & $1(2)$ \\
\hline Padres hipertensos & $1(2)$ \\
\hline \multicolumn{2}{|l|}{ Antecedentes patológicos } \\
\hline Negados & $22(44)$ \\
\hline Hipertensión & $11(22)$ \\
\hline Hipertensivo/diabetes & $6(12)$ \\
\hline Diabetes & $5(10)$ \\
\hline Arritmia cardiaca & $2(4)$ \\
\hline Colecistectomía & $1(2)$ \\
\hline Crisis asmáticas & $1(2)$ \\
\hline Crisis convulsivas & $1(2)$ \\
\hline
\end{tabular}

* La edad es expresada en años cumplidos desde el nacimiento en promedio y desviación estándar. 
lengua saburral (62.5\%), edentulismo (12.5\%), úlceras (43.8\%), necrosis $(12.5 \%)$, xerostomía $(43.8 \%)$ y caries dental (75\%), mientras que los sujetos con antecedentes de trauma craneoencefálico mostraron distribuciones mayores en las condiciones o alteraciones bucodentales como periodontitis $(66.7 \%)$, movilidad severa $(25 \%)$, irritación de tejidos (12.7\%), edentulismo parcial (50\%) y cálculo dental (66.7\%). Por otro lado, las asociaciones estadísticas encontradas para los sujetos con evento cerebral vascular y los sujetos con trauma craneoencefálico fueron antecedente de tabaquismo, hipertensión arterial, hipertensión arterial y diabetes mellitus, gingivitis, perio-

Tabla 2: Distribución de alteraciones bucodentales $(\mathrm{N}=50)$.

\begin{tabular}{lc} 
& n (\%) \\
\hline Caries & $38(76)$ \\
Lengua saburral & $32(64)$ \\
Cálculo dental & $29(58)$ \\
Xerostomía & $26(52)$ \\
Periodontitis & $22(44)$ \\
Úlceras & $18(36)$ \\
Edentulismo parcial & $17(34)$ \\
Gingivitis & $9(18)$ \\
Edéntulo & $7(14)$ \\
Movilidad severa & $5(10)$ \\
Fractura & $4(8)$ \\
Necrosis & $4(8)$ \\
Irritación de tejidos & $4(8)$ \\
\hline
\end{tabular}

dontitis, movilidad severa y úlceras. De esta forma, los sujetos con evento cerebral vascular se encuentran estadísticamente asociados con la presencia de tabaquismo (56.3\%), hipertensión arterial (50\%), hipertensión arterial y diabetes mellitus (37.5\%), gingivitis (37.5\%) y las úlceras $(43.8 \%)$, en comparación con pacientes con trauma craneoencefálico (0-8.3\%); por lo tanto, los sujetos con antecedente de trauma craneoencefálico tuvieron asociaciones directamente con la presencia de periodontitis $(66.7 \%)$ y movilidad severa ( $25 \%)$ que aquellos pacientes con evento cerebral vascular (10 y $0 \%$, respectivamente).

\section{DISCUSIÓN}

En el presente estudio se encontró al evento cerebral vascular y al traumatismo craneoencefálico como los principales motivos de hospitalización e intubación, determinando que las principales alteraciones bucales fueron la caries dental, lengua saburral, cálculo dental, xerostomía, periodontitis, úlceras, entre otras. En particular, el evento cerebral vascular se asoció significativamente con tabaquismo, hipertensión arterial, hipertensión arterial con diabetes mellitus, gingivitis y úlceras, mientras que el traumatismo craneoencefálico sólo se asoció estadísticamente con periodontitis. Lo anterior parece indicar que el motivo de hospitalización/intubación juega un rol importante en las alteraciones que los casos tienen dental y sistémicamente, en especial hábitos no patológicos, de higiene bucal y condiciones sistémicas crónico-degenerativas. Estos resultados deberían ser compartidos con el personal de salud, sobre todo con aquéllos que procuren el cuidado de los pacientes con intubación para determi-
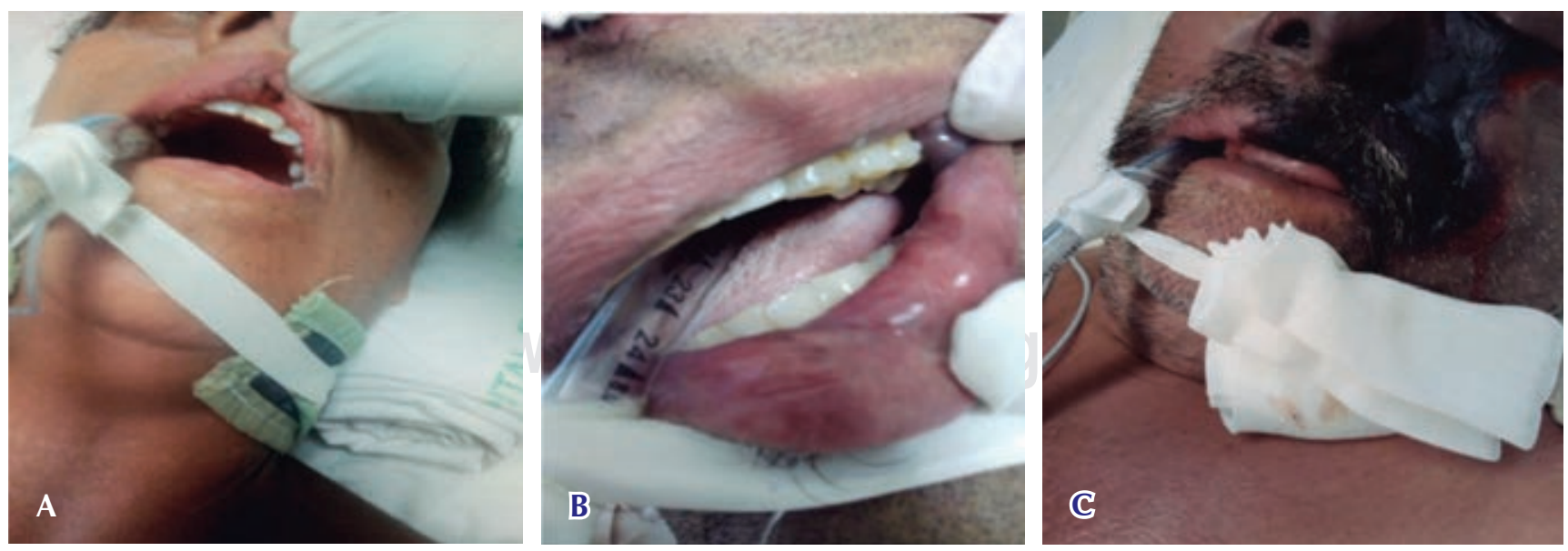

Figura 1: A) Úlcera por presión en labio superior. B) Lengua saburral. C) Necrosis. 
Tabla 3: Distribución de alteraciones generales y bucales de pacientes con evento cerebral vascular y trauma craneoencefálico.

\begin{tabular}{lccc} 
& $\begin{array}{c}\text { Evento cerebral vascular (N = 16) } \\
\mathbf{n}(\mathbf{\%})\end{array}$ & $\begin{array}{c}\text { Trauma craneoencefálico (N = 12) } \\
\mathbf{n}(\%)\end{array}$ & $\mathbf{p}$ \\
\hline Personales & & & $0.004^{*}$ \\
Tabaquismo & $9(56.3)$ & $0(0.0)$ & 0.089 \\
Etilismo & $1(6.3)$ & $4(33.3)$ & 0.357 \\
Tabaquismo/etilismo & $2(12.5)$ & $3(25.0)$ & $0.004^{*}$ \\
Patológicos & & & $0.021^{*}$ \\
Hipertensión & $8(50.0)$ & $0(0.0)$ & 0.611 \\
Diabético & $0(0.0)$ & $0(0.0)$ & 0.565 \\
Hipertensión/diabético & $6(37.5)$ & $0(0.0)$ & 0.609 \\
Alteraciones bucales & & & 0.672 \\
Edéntulo & $2(12.5)$ & $1(8.3)$ & $0.021^{*}$ \\
Lengua saburral & $10(62.5)$ & $1(8.3)$ & $0.014^{*}$ \\
Xerostomía & $7(43.8)$ & $5(41.7)$ & 0.067 \\
Caries & $12(75.0)$ & $9(75.0)$ & $0.048^{*}$ \\
Gingivitis & $6(37.5)$ & $0(0.0)$ & 0.611 \\
Periodontitis & $3(10.0)$ & $8(66.7)$ & 0.389 \\
Movilidad severa & $0(0.0)$ & $3(25.0)$ & 0.611 \\
Úlceras & $7(43.8)$ & $1(8.3)$ & 0.167 \\
Necrosis & $2(12.5)$ & $1(8.3)$ & 0.207 \\
Irritación de tejidos & $1(6.3)$ & $2(12.7)$ & $1(8.3)$ \\
Fractura & $2(12.5)$ & $6(50.0)$ & $8(66.7)$ \\
Edentulismo parcial & $4(25.0)$ & & \\
Cálculo dental & $7(43.8)$ & & \\
\hline
\end{tabular}

* Diferencia estadísticamente significativa $(\mathrm{p}<0.05)$

nar el diagnóstico y prevención oportuna de afecciones bucales principalmente.

Las complicaciones que se presentan durante el proceso de intubación han sido descritas en la literatura como un factor negativo que afecta la recuperación del paciente. ${ }^{6}$ En un estudio elaborado en 2015 por anestesiólogos, se evaluaron los factores de riesgo que ocasionan lesiones bucodentales durante el proceso de intubación, $80 \%$ de ellos concluyeron que las lesiones dentales son ocasionadas al momento de realizar la intubación. ${ }^{7}$ Existen algunas patologías bucodentales que podrían ser factores de riesgo y aumentar la presencia de lesiones durante la intubación como la caries, periodontitis y restauraciones existentes.

En un estudio realizado en el Servicio de Urgencias de un Hospital en México donde evaluaron a 150 pacientes con intubación, encontraron que el evento cerebral vascular fue la indicación más frecuente en requerir asistencia ventilatoria. Por otra parte, observaron que en su población de pacientes jóvenes el motivo de intubación era ocasionado por traumatismo craneoencefálico. ${ }^{8}$ En nuestra investigación, el evento cerebral vascular fue el motivo más frecuente de hospitalización e intubación, esto podría estar relacionado por la tasa de hipertensión arterial y diabetes mellitus no controlada en el estado de Chihuahua; a su vez, el tabaquismo es uno de los hábitos más comunes en la población, siendo éstos los factores que aumentan el riesgo para desencadenar enfermedades cerebrovasculares. ${ }^{9-11}$ El siguiente motivo de hospitalización más frecuente fue el traumatismo craneoencefálico, según el INEGI el estado de Chihuahua se encuentra entre los tres primeros lugares en los índices de accidentes automovilísticos, lo cual podría ser un factor en la frecuencia en este motivo de hospitalización.

La caries y cálculo dental fueron las alteraciones más frecuentes entre los grupos; si bien estas afecciones ya estaban presentes previo a la intubación, la falta de 
higiene en las unidades de cuidados intensivos podría aumentar el riesgo de desarrollar placa bacteriana y elevar la severidad de estas lesiones bucodentales. Un estudio realizado en 2001 reportó a las lesiones gingivales y labiales como las más frecuentes durante el proceso de intubación. ${ }^{12}$ En nuestro trabajo, los pacientes con EVC se asociaron significativamente con gingivitis y los pacientes con TCE fueron asociados con periodontitis, si bien no se saben las patologías bucodentales presentes en los pacientes previo a la hospitalización, está documentado que a partir de las 48 horas que el paciente es sometido al proceso de intubación sufre un cambio en su microbiota oral, además, si la placa dental no es retirada, podría generar el desarrollo de gingivitis en menos de 10 días y progresar a enfermedades dentales más graves como la periodontitis. ${ }^{13}$ Un estudio realizado en 2007 encontró que las bacterias que colonizan la cavidad bucal se encuentran en un $70 \%$ en el biofilm dental, $63 \%$ en la lengua y $73 \%$ en el tubo utilizado para la ventilación. ${ }^{14}$ A su vez, el tubo endotraqueal generalmente ocasiona lesiones en la mucosa y la severidad podría variar de acuerdo con el tiempo de intubación y a la presión que ejerce el aditamento en los tejidos, esto podría desarrollar úlceras ocasionadas por la compresión. ${ }^{15}$ Los pacientes con EVC se asociaron significativamente con la presencia de úlceras, esto podría estar relacionado con la condición sistémica crónico-degenerativa de los pacientes como la diabetes mellitus, la cual está asociada al desarrollo de múltiples complicaciones orales, ya que reduce la resistencia inmunológica de los tejidos, así como la síntesis y metabolismo de colágeno. ${ }^{16}$

Por otra parte, lesiones bucales como la lengua saburral y xerostomía también se encontraron entre las alteraciones bucales más frecuentes; la lengua saburral, que se caracteriza por la presencia de restos de alimentos, placa dental, restos de bacterias y células descamadas, ${ }^{17}$ se encontró en mayor frecuencia en pacientes con EVC, dentro de las principales causas relacionadas con la formación de la capa de saburra en la lengua se encuentra la deficiencia en la higiene. ${ }^{18}$ Por otro lado, la presencia de xerostomía fue observada en ambos grupos, lo que podría suponer que la presencia de las sondas endotraqueales causan que inevitablemente se mantenga la boca abierta, facilitando la sequedad y disminución de saliva en los pacientes. ${ }^{13}$ Dentro de la distribución de las alteraciones bucodentales se observaron en menor frecuencia edentulismo, movilidad severa y fractura, la mayoría de estas lesiones son provocadas al momento de la intubación, durante la entrada del laringoscopio, ${ }^{3}$ la cual conlleva a las lesiones de dientes, labios, encías y otros tejidos orales ocasionados por la fuerza de compresión que se aplica. ${ }^{3,12}$ Es por lo que hoy en día se recomienda una revisión de la cavidad oral cada ocho horas según la Asociación Norteamericana de Cuidados Intensivos en Enfermería. ${ }^{19}$

Si bien hay numerosos trabajos en la literatura donde se estudia la distribución de afecciones bucodentales en pacientes intubados, hay pocos estudios sobre la asociación entre estas afecciones y el motivo de hospitalización, sobre todo en el estado de Chihuahua. Esta información podría ser analizada por personal de hospitales a cargo de los pacientes intubados, ya que podría mejorar la salud y disminuir el desarrollo de alteraciones bucodentales durante el proceso de asistencia ventilatoria.

Este trabajo tiene como limitante la población de estudio, futuras investigaciones con un mayor número de pacientes deberían llevarse a cabo para evaluar las alteraciones bucodentales ocasionadas por la intubación, así como posibles estrategias para poder evitarlas.

\section{CONCLUSIONES}

Podemos concluir que la intubación es un procedimiento que, por la presencia de los tubos de ventilación, ayuda a generar situaciones que actúan como factores para desarrollar lesiones en la mucosa oral, órganos dentales, lengua y tejidos adyacentes. Sin embargo, el motivo de hospitalización y las condiciones sistémicas preexistentes juegan un factor de riesgo importante para desarrollar lesiones bucales específicas antes y durante el periodo de intubación.

\section{AGRADECIMIENTOS}

Los autores agradecen al Hospital General de Ciudad Juárez, Chihuahua del Instituto Chihuahuense de Salud y al Departamento de Estomatología por las facilidades en la realización del estudio.

\section{REFERENCIAS}

1. Buitrago CQ. Lesiones orales en el manejo de la vía aérea. Rev Colomb Anestesiol. 2008; 36 (2): 121-132. doi: 10.1016/s01203347(08)62005-5.

2. Ostabal Artigas MI. La intubación endotraqueal. Medicina Integral. 2002; 39 (8): 335-342.

3. Vogel J, Stübinger S, Kaufmann M, Krastl G, Filippi A. Dental injuries resulting from tracheal intubation--a retrospective study. Dent Traumatol. 2009; 25 (1): 73-77. doi: 10.1111/j.16009657.2008.00670.x.

4. White A, Kander PL. Anatomical factors in difficult direct laryngoscopy. Br J Anaesth. 1975; 47 (4): 468-474. doi: 10.1093/ bja/47.4.468. 
5. Schmidt BL. Dental complications. In: Eisele D, Smith R. Complications in head and neck surgery with $C D$ image bank. Elsevier Inc.; 2009. pp. 267-277. doi: 10.1016/B978-1416042204.50028-6.

6. Pomposo EMA, Hurtado RIC, Jiménez RA, Barriga FP, Bravo SJCG. Complicaciones postextubación asociadas con la presión de inflado del globo del tubo endotraqueal. An Med Asoc Med Hosp ABC. 2014; 59 (2): 115-119.

7. Darawade DA, Dubey A, Gondhalekar R, Dahapute S, Deshmukh $\mathrm{SB}$, Darawade AD. Assessment of the risk factors for oro-dental injuries to occur during general anesthesia and measures taken by anesthesiologist to prevent them. J Int Oral Health. 2015; 7 (7): 77-79.

8. Chavarría-Islas RA, Robles BLA, Loria CJ, Rocha LJM. Complicaciones agudas por intubación orotraqueal en un Servicio de Urgencias. Arch Med Urg Mex. 2012; 4 (1): 20-25.

9. Fernández Cherkásova L, Díaz Zayas N, Guevara Rodríguez IM. Estudio de factores de riesgo de la enfermedad cerebrovascular en el Policlínico Docente "Doctor Carlos J. Finlay". Rev Cubana Med Gen Integr [Internet]. 2004; 20 (1). Disponible en: http://scielo.sld.cu/scielo.php?script=sci_arttext\&pid=S0864$21252004000100005 \& \operatorname{lng}=\mathrm{es}$

10. Kissela BM, Khoury JC, Alwell K et al. Age at stroke: temporal trends in stroke incidence in a large, biracial population. Neurology. 2012; 79 (17): 1781-1787. doi: 10.1212/WNL.0b013e318270401d.

11. Torres Damas EL. Factores de riesgo aterogénico en población adulta venezolana. Rev Cubana Angiol Cir Vasc. 2016; 17 (1).

12. Fung BK, Chan MY. Incidence of oral tissue trauma after the administration of general anesthesia. Acta Anaesthesiol Sin. 2001; 39 (4): 163-167.

13. Johnstone L, Spence D, Koziol-McClain J. Oral hygiene care in the pediatric intensive care unit: practice recommendations. Pediatr Nurs. 2010; 36 (2): 85-96; quiz 97.

14. Oliveira LCBS, Carneiro PPM, Fischer RG, Tinoco BEM. A presença de Patógenos Respiratórios no Biofilme Bucal de Paciente com
Pneumonia Nosocomial. Rev Bras Ter Intens,. 2007; 19 (4): 428433. doi: 10.1590/s0103-507x2007000400004.

15. Santos PM, Afrassiabi A, Weymuller EA Jr. Prospective studies evaluating the standard endotracheal tube and a prototype endotracheal tube. Ann Otol Rhinol Laryngol. 1989; 98 (12 Pt 1): 935-940. doi: 10.1177/000348948909801204.

16. McKenna SJ. Dental management of patients with diabetes. Dent Clin North Am. 2006; 50 (4): 591-606. doi: 10.1016/j. cden.2006.06.008.

17. Guzmán Marín B, Calero JA. Principales hallazgos clínicos en la lengua en una población de Cali. Rev Estomat. 2005; 13 (1): 5-12.

18. Tejada GA. Prevalencia de lesiones bucales en tejido blando encontradas en la Clínica de Estomatología de la Facultad de Odontología de la Universidad de los Andes. Periodo 2015-2018. Rev ADM. 2020; 77 (1): 11-16. doi: 10.35366/OD201C.

19. Jones $\mathrm{H}$. Oral care in intensive care units: a literature review. Spec Care Dent. 2005; 25 (1): 6-11. doi: 10.1111/j.1754-4505.2005. tb00366.x.

Conflicto de intereses: Los autores declaran no tener ningún conflicto de intereses.

Aspectos éticos: El estudio fue aprobado por el jefe del Departamento de Enseñanza de la Institución Hospital General de Ciudad Juárez, Chihuahua del Instituto Chihuahuense de Salud.

Financiamiento: Sin financiamiento.

\section{Correspondencia:}

Dr. León Francisco Espinosa Cristóbal

E-mail: leon.espinosa@uacj.mx 